\title{
Role of Fibroblast Growth Factor Receptor 2 in Pancreatic Cancer: Potential Target for New Therapeutic Approach?
}

Enrica Vescarelli ${ }^{1}$, Simona Ceccarelli ${ }^{1}$ and Antonio Angeloni ${ }^{*}$

${ }^{1}$ Department of Experimental Medicine, Sapienza University of Rome, Viale Regina Elena, Rome, Italy

${ }^{2}$ Department of Molecular Medicine, Sapienza University of Rome, Viale Regina Elena, Rome, Italy

*Corresponding author: Antonio Angeloni, Department of Molecular Medicine, Sapienza University of Rome, Viale Regina Elena 324, 00161 Rome, Italy, Tel: +390649973012; E-mail: antonio.angeloni@uniroma1.it

Rec date: Oct 30, 2015; Acc date: Nov 20, 2015; Pub date: Nov 23, 2015

Copyright: $\odot 2015$ Vescarelli E, et al. This is an open-access article distributed under the terms of the Creative Commons Attribution License, which permits unrestricted use, distribution, and reproduction in any medium, provided the original author and source are credited.

\begin{abstract}
Fibroblast growth factors and their receptors play a key role in cell proliferation, migration and differentiation. Fibroblast growth factor receptor 2 (FGFR2) is involved in carcinogenesis and its altered expression has been shown in several tumors, such as breast, thyroid and pancreatic cancer. The two isoforms of FGFR2 gene, FGFR2Illb (also known as KGFR) and FGFR2-IIlc have been shown to exert differential roles in pancreatic cancer. FGFR2IIIc supports pancreatic cell proliferation, while overexpression of FGFR2-IIIb is correlated to major invasion and metastasis formation. This review focuses on the role of FGFR2 signaling in pancreatic adenocarcinoma and the potential use of FGFR2 tissutal expression as a predictive and/or prognostic marker. Moreover, it will discuss about the potential use of strategies for FGFR2 signaling inhibition in the treatment of pancreatic cancer.
\end{abstract}

Keywords: Pancreatic adenocarcinoma; FGFR2; FGFR2-iiib; FGFR2-Iiic; Therapeutic strategies

\section{Introduction}

FGFRs are a family of tyrosine kinase receptors consisting of four members (FGFR1-4). These receptors are characterized by two or three immunoglobulin-like extracellular domains, an intracellular tyrosine kinase domain, and a carboxyl-terminus domain [1]. They are involved in regulating cell proliferation, migration and differentiation during vertebrate development, as well as in response to injury and tissue repair [2]. In this review, we focus our attention on the FGFR2 gene, which is subjected to an alternative splicing that generates two isoforms, FGFR2-IIIb, predominantly expressed on epithelial cells, and FGFR2-IIIc, detected in mesenchymal cells. FGFR2-IIIb has a role in the control of epithelial growth and differentiation, while FGFR2-IIIc receptor is involved in the proliferation of mesenchymal cells. The two isoforms show a different affinity for ligands; in particular, FGFR2-IIIb isoform binds with high affinity to KGF (also known as FGF7), FGF10 and FGF22, and with low affinity to FGF1 and FGF3, while the FGFR2-IIIc isoform binds to FGF1, FGF2, FGF4, FGF5, FGF6, FGF8, FGF9, FGF16, FGF17, FGF18, FGF20, FGF21 and FGF23 [3].

Some studies proposed that a shift between splicing isoforms may be involved in tumor progression. For example, a switch between FGFR2-IIIb and FGFR2-IIIc isoform in rat prostate and bladder cancer models might contribute to alter the balance between epithelial and mesenchymal cells and to promote epithelial-mesenchymal transition $[4,5]$. However, FGFR2-IIIb isoform re-expression in prostate and bladder cancer cell lines resulted in growth suppression in vitro and in decreased tumor formation in vivo [6-8].

Up- or down-modulation of one or both FGFR2 isoforms has been shown in several types of cancer, for example in thyroid, breast, lung, stomach and pancreas cancer [9-12]. Genetic mutations of FGFR2 have been also observed, and they are correlated with cancer susceptibility or poor prognosis. In breast cancer, single nucleotide polymorphisms (SNPs) located within intron 2 of FGFR2 gene have been associated to increased risk of cancer $[13,14]$. Missense mutations of FGFR2 have been observed in breast, gastric and lung cancer. Some of these mutations can alter ligand-receptor specificity or determine a ligand-independent FGFR2 activation [15]. In the present work, we discuss the role of FGFR2 signaling pathway in pancreatic cancer and the potential use of FGFR2 inhibitors as anti-cancer therapies.

\section{Role of FGFR2 in Pancreatic Adenocarcinoma}

Pancreatic ductal adenocarcinoma (PDAC) is an aggressive human malignancy with high mortalities among all malignancies [16]. Several growth factors and their receptors are overexpressed in PDAC, such as transforming growth factor-a, epidermal growth factor receptor, FGFs, and FGFRs [17]. Several FGFs are overexpressed in pancreatic cancer [18] and their altered expression has been linked to increased pancreatic cancer cell motility, proliferation, invasion and metastatic potential $[19,20]$. Expression levels of FGFR1 and FGFR2 have been reported to be involved in pancreatic cancer development. FGFR1 overexpression is implicated in growth of pancreatic cancer cells [21,22], while increase of FGFR2 phosphorylation is linked with enhanced pancreatic cell proliferation, invasion and tumor angiogenesis [23].

Differential expression of FGFR2-IIIb and its ligand has been shown in different pancreatic cell types, such as acinar, ductal and islet cells [24]. We also confirmed these results by immunohistochemical staining on a tissue array of 40 cases of ductal adenocarcinoma, grade 1-3, with a home-made monoclonal antibody against FGFR2-IIIb [25]. Furthermore, several studies show concomitant overexpression of FGFR2-IIIb and its ligand KGF in PDAC [26,27], which is correlated with high expression of vascular endothelial growth factor-A (VEGF$A)$, venous invasion, and poor prognosis [28]. In metastatic pancreatic cancer cell lines, the upregulation of KGF secretion is linked with pancreatic progression and metastatic formation, suggesting that the 
altered expression of KGF and FGFR2-IIIb may be involved in early signals in the progression of pancreatic cancer [29].

As previously reported, normal pancreatic cells lack FGFR2-IIIc, while enhanced expression of the latter has been detected in pancreatic cancer cell lines and PDAC tissues, boosting proliferative ability in vivo [30].

Several studies have shown a correlation between FGFR2 gene amplification or single nucleotide polymorphism in intron 2 with cancer progression [11,12,31]. FGFR2 amplification has been associated to poorly differentiate gastric cancer [32]. Notably, an amplification of FGFR2 was also detected in pancreatic cancer based on cell line and xenograft aberration profiles originated from genomewide analysis [33]. Most importantly, FGFR2 inhibition by means of specific shRNA, leads to cell proliferation, migration and invasion [34].

Soluble factors released from tumor stroma have been shown to play a role in tumor growth. For example, cell-derived factor-1 (SDF-1/ CXCL12) released by fibroblasts can promote cancer cells proliferation, while CXCR4 is released in several types of malignancies, such as breast cancer [35] and pancreatic cancer [36,37]. Also FGF10, produced by fibroblasts, has been demonstrated to induce cell migration and invasion in pancreatic cancer cells [19], while FGF7 is able to promote pancreatic cell proliferation. Nomura et al., have investigated the role of FGF10/FGFR2 pathway in pancreatic cancer. Their results demonstrated that FGF10 induces cell migration and invasion, thus influencing the expression of genes related to cell mobility, such as membrane type 1-matrix metalloproteinase (MT1MMP) and transforming growth factor beta (TGF- $\beta 1$ ). In particular, mRNA expression of MT1-MMP and TGF- $\beta 1$ were upregulated by FGF10 in CFPAC-1 cells.

\section{FGF/FGFR Signaling as a Therapeutic Target}

Pancreatic adenocarcinoma is characterized by high mortality, incidence of metastatic disease and rapid progression. It is very difficult to obtain an early diagnosis, and in the majority of cases at the time of diagnosis patients just show several metastases, with an unfavorable prognosis. Therefore, new therapies for pancreatic cancer are extremely necessary. To detect new molecular mechanisms at the basis of the onset and progression of this cancer might improve its diagnosis and therapy.

Growth factors and their receptors have a role in several events that occur in tumorigenesis [38]. Since FGFR, in particular, is involved in cancer progression and invasion, its signaling may represent a therapeutic target for anticancer therapy. Several therapeutical approaches against FGFR are now available. Small molecular tyrosine kinase inhibitors with anti-angiogenic activity against VEGFR and PDGFR are currently in preclinical or clinical trial. Dovitinib (TKI-258), an inhibitor of FGFR1, FGFR2 and FGFR3, showed antitumor activity in FGFR-amplified breast cancer [39]. This inhibitor has been also evaluated in bladder cancer and relapsed multiple myeloma cases. An alternative therapeutical strategy is based on the use of neutralizing antibodies. This method promises a high specificity against the molecule of interest, thus allowing the development of targeted therapies. Some of these monoclonal antibodies, just approved by FDA, are nowadays used as anticancer monotherapy or in combination with standard chemotherapy. For example, anti-EGFR antibodies, such as Cetiximab and Panitumumab, are now used in the treatment of colon cancer, while the anti-HER2 antibody Trastuzumab is currently administered to breast cancer and gastric cancer patients in combination with chemotherapy. Also FGFs and FGFRs became a relevant target for the development of specific neutralizing antibodies. Specific antibodies against FGFR1/FGFR3 were tested in bladder and myeloma cancer with successful results [40,41]. Another approach consists in the use of FGF ligand traps that prevent FGF binding with its receptor.

FGFR2 has a key role in the establishment of cancer, so it represents a potential target for cancer treatment. Monoclonal antibodies for this receptor have been produced. Bai et al., created a specific antibody against FGFR2-IIIb isoform that is able to suppress ligand induced phosphorylation [42]. As concerning pancreatic cancer therapy, the use of monoclonal antibodies against growth factors has been evaluated. Phase I clinical trials showed the feasibility of Panitumumab, a monoclonal antibody against epidermal growth factor (EGFR), for the treatment of patients with locally advanced pancreatic cancer (LAPC). The combined use of panitumumab, at a maximum tolerated dose of $1.5 \mathrm{mg} / \mathrm{kg}$, with gemcitabine-based chemoradiotherapy, can be safely and effectively used in LAPC patients [43]. In PDAC, not only cancer cells but also tumor microenvironment contributes to the initiation, progression, and metastasis of tumor. Therefore, pancreatic cancer is extremely complex, and the use of single monoclonal antibody therapy is not a successful therapeutic strategy. Nevertheless, the discovery of new predictive and response biomarkers is necessary to develop new therapeutic approaches. In light of these considerations, FGFR2 might be a marker for pancreatic cancer and its investigation is needed to evaluate its potential use as a target for therapy.

\section{Limitations of anti-FGFR2 Therapeutic Approaches}

FGFR2 plays a relevant role in pancreatic cancer and it might represent a potential marker for new therapeutic strategies. In particular, in this review we focused our attention on FGFR2-IIIb/KGF signaling, which is involved in pancreatic cancer progression, invasion and metastasis formation. A therapeutic approach against FGFR2-IIIb isoform could be useful, but the lack of highly specific drug against FGFR2-IIIb is the major limitation of this approach. In fact, the use of pan-FGFR inhibitors might influence also other signaling. Nowadays, clinical trials on tyrosine kinase inhibitors direct against FGFR1/2/3 are available, but these inhibitors do not target all activating mutation. Therefore, studies to determine which aberration plays a role in tumorigenesis are required in order to understand the implication of genomic alteration.

GP369 is the only antibody used in vitro and in vivo to suppress IIIb isoform in amplified human breast and gastric cancer [42]. This antibody is efficient only in cancers with FGFR2 amplification, so it would be necessary to evaluate FGFR2-IIIb expression and type of mutation before administration of FGFR2-IIIb inhibitor to pancreatic cancer patients. Therefore, FGFR2 evaluation should be introduced in routine diagnosis. For this reason, the use of therapeutic approach base on FGFR2 might have several limitations due to the need for expensive genetic analyses.

\section{Conclusions}

Pancreatic adenocarcinoma is characterized by high mortality and poor prognosis due late diagnosis. The lack of predictive and prognostic markers supports the aggressive nature of pancreatic tumors. Several growth factors and their receptors have been shown to be involved in cancer onset and progression, and the investigation of 
their potential as predictive and prognostic markers might be useful to ameliorate cancer diagnosis.

FGFRs family has an important role during physiological and pathological processes, and also in carcinogenesis. FGFR2 altered expression was observed in different types of cancer, including pancreatic ductal adenocarcinoma. The overexpression of this receptor is associated to major invasion, cell proliferation and metastasis formation; therefore FGFR2 up regulation might be considered an early step in the development of pancreatic cancer. For this reason, FGFR2 might represent a potential marker for diagnostic and prognostic evaluation of pancreatic cancer.

The identification of novel molecules involved in pancreatic tumor progression might result in the development of promising therapeutic strategies, thus increasing the available anticancer agents and treatment opportunities. Nowadays, different therapeutic approaches have been produced against FGFR2, and the current information about the relationship between this receptor and pancreatic cancer clearly indicate that the inhibition of this signalling pathway could find an application also in the treatment of PDAC.

\section{References}

1. Eswarakumar VP, Lax I, Schlessinger J (2005) Cellular signaling by fibroblast growth factor receptors. Cytokine Growth Factor Rev 16 139-149.

2. Marchese C, Chedid M, Dirsch OR, Csaky KG, Santanelli F, et al. (1995) Modulation of keratinocyte growth factor and its receptor in reepithelializing human skin. J Exp Med 182: 1369-1376.

3. Holzmann K, Grunt T, Heinzle C, Sampl S, Steinhoff H, et al. (2012) Alternative Splicing of Fibroblast Growth Factor Receptor IgIII Loops in Cancer. J Nucleic Acids 2012: 950508.

4. Savagner P, Valles AM, Jouanneau J, Yamada KM, Thiery JP (1994) Alternative splicing in fibroblast growth factor receptor 2 is associated with induced epithelial-mesenchymal transition in rat bladder carcinoma cells. Mol Biol Cell 5: 851-862.

5. Oltean S, Sorg BS, Albrecht T, Bonano VI, Brazas RM, et al. (2006) Alternative inclusion of fibroblast growth factor receptor 2 exon IIIc in Dunning prostate tumors reveals unexpected epithelial mesenchymal plasticity. Proc Natl Acad Sci U S A 103: 14116-14121.

6. Matsubara A, Kan M, Feng S, McKeehan WL (1998) Inhibition of growth of malignant rat prostate tumor cells by restoration of fibroblast growth factor receptor 2. Cancer Res 58: 1509-1514.

7. Ricol D, Cappellen D, El Marjou A, Gil-Diez-de-Medina S, Girault JM, et al. (1999) Tumour suppressive properties of fibroblast growth factor receptor 2-IIIb in human bladder cancer. Oncogene 18: 7234-7243.

8. Yasumoto H, Matsubara A, Mutaguchi K, Usui T, McKeehan WL (2004) Restoration of fibroblast growth factor receptor suppresses growth and tumorigenicity of malignant human prostate carcinoma PC-3 cells. Prostate 61: 236-242.

9. St Bernard R, Zheng L, Liu W, Winer D, Asa SL, et al. (2005) Fibroblast growth factor receptors as molecular targets in thyroid carcinoma. Endocrinology 146: 1145-1153.

10. Finch PW, Rubin JS (2006) Keratinocyte growth factor expression and activity in cancer: implications for use in patients with solid tumors. J Natl Cancer Inst 98: 812-824.

11. Katoh M (2008) Cancer genomics and genetics of FGFR2 (Review). Int J Oncol 33: 233-237.

12. Katoh Y, Katoh M (2009) FGFR2-related pathogenesis and FGFR2targeted therapeutics (Review). Int J Mol Med 23: 307-311.

13. Easton DF, Pooley KA, Dunning AM, Pharoah PD, Thompson D, et al. (2007) Genome-wide association study identifies novel breast cancer susceptibility loci. Nature 447: 1087-1093.
14. Hunter DJ, Kraft P, Jacobs KB, Cox DG, Yeager M, et al. (2007) A genome-wide association study identifies alleles in FGFR2 associated with risk of sporadic postmenopausal breast cancer. Nat Genet 39: 870-874.

15. Pollock PM, Gartside MG, Dejeza LC, Powell MA, Mallon MA, et al. (2007) Frequent activating FGFR2 mutations in endometrial carcinomas parallel germline mutations associated with craniosynostosis and skeletal dysplasia syndromes. Oncogene 26: 7158-7162.

16. Siegel R, Naishadham D, Jemal A (2012) Cancer statistics, 2012. CA Cancer J Clin 62: 10-29.

17. Kornmann M, Beger HG, Korc M (1998) Role of fibroblast growth factors and their receptors in pancreatic cancer and chronic pancreatitis. Pancreas 17: 169-175.

18. Mahadevan D, Von Hoff DD (2007) Tumor-stroma interactions in pancreatic ductal adenocarcinoma. Mol Cancer Ther 6: 1186-1197.

19. Nomura S, Yoshitomi H, Takano S, Shida T, Kobayashi S, et al. (2008) FGF10/FGFR2 signal induces cell migration and invasion in pancreatic cancer. Br J Cancer 99: 305-313.

20. Yi ES, Yin S, Harclerode DL, Bedoya A, Bikhazi NB, et al. (1994) Keratinocyte growth factor induces pancreatic ductal epithelial proliferation. Am J Pathol 145: 80-85.

21. Liu Z, Neiss N, Zhou S, Henne-Bruns D, Korc M, et al. (2007) Identification of a fibroblast growth factor receptor 1 splice variant that inhibits pancreatic cancer cell growth. Cancer Res 67: 2712-2719.

22. Chen G, Tian X, Liu Z, Zhou S, Schmidt B, et al. (2010) Inhibition of endogenous SPARC enhances pancreatic cancer cell growth: modulation by FGFR1-III isoform expression. Br J Cancer 102: 188-195.

23. Wesche J, Haglund K, Haugsten EM (2011) Fibroblast growth factors and their receptors in cancer. Biochem J 437: 199-213.

24. Ishiwata T, Friess H, Büchler MW, Lopez ME, Korc M (1998) Characterization of keratinocyte growth factor and receptor expression in human pancreatic cancer. Am J Pathol 153: 213-222.

25. Ceccarelli S, Bei R, Vescarelli E, D'Amici S, di Gioia C, et al. (2014) Potential prognostic and diagnostic application of a novel monoclonal antibody against keratinocyte growth factor receptor. Mol Biotechnol 56 939-952.

26. Yamanaka Y, Friess H, Buchler M, Beger HG, Uchida E, et al. (1993) Overexpression of acidic and basic fibroblast growth factors in human pancreatic cancer correlates with advanced tumor stage. Cancer Res 53 : $5289-5296$.

27. Siddiqi I, Funatomi H, Kobrin MS, Friess H, Büchler MW, et al. (1995) Increased expression of keratinocyte growth factor in human pancreatic cancer. Biochem Biophys Res Commun 215: 309-315.

28. Cho K, Ishiwata T, Uchida E, Nakazawa N, Korc M, et al. (2007) Enhanced expression of keratinocyte growth factor and its receptor correlates with venous invasion in pancreatic cancer. Am J Pathol 170: 1964-1974.

29. Zang XP, Lerner M, Brackett D, Pento JT (2009) Influence of KGF on the progression of pancreatic cancer. Anticancer Res 29: 3417-3420.

30. Ishiwata T, Matsuda Y, Yamamoto T, Uchida E, Korc M, et al. (2012) Enhanced expression of fibroblast growth factor receptor 2 IIIc promotes human pancreatic cancer cell proliferation. Am J Pathol 180: 1928-1941.

31. Katoh M (2009) FGFR2 abnormalities underlie a spectrum of bone, skin, and cancer pathologies. J Invest Dermatol 129: 1861-1867.

32. Hattori $\mathrm{Y}$, Itoh $\mathrm{H}$, Uchino S, Hosokawa K, Ochiai A, et al. (1996) Immunohistochemical detection of $\mathrm{K}$-sam protein in stomach cancer. Clin Cancer Res 2: 1373-1381.

33. Nowak NJ, Gaile D, Conroy JM, McQuaid D, Cowell J, et al. (2005) Genome-wide aberrations in pancreatic adenocarcinoma. Cancer Genet Cytogenet 161: 36-50.

34. Matsuda Y, Yoshimura H, Suzuki T, Uchida E, Naito Z, et al. (2014) Inhibition of fibroblast growth factor receptor 2 attenuates proliferation and invasion of pancreatic cancer. Cancer Sci 105: 1212-1219.

35. Orimo A, Gupta PB, Sgroi DC, Arenzana-Seisdedos F, Delaunay T, et al. (2005) Stromal fibroblasts present in invasive human breast carcinomas 
Citation: Vescarelli E, Ceccarelli S, Angeloni A (2015) Role of Fibroblast Growth Factor Receptor 2 in Pancreatic Cancer: Potential Target for New Therapeutic Approach?. Pancreat Disord Ther 5: 164. doi:10.4172/2165-7092.1000164

Page 4 of 4

promote tumor growth and angiogenesis through elevated SDF-1/ CXCL12 secretion. Cell 121:335-348.

36. Koshiba T, Hosotani R, Miyamoto Y, Ida J, Tsuji S, et al. (2000) Expression of stromal cell-derived factor 1 and CXCR4 ligand receptor system in pancreatic cancer: a possible role for tumor progression. Clin Cancer Res 6: 3530-3535.

37. Marchesi F, Monti P, Leone BE, Zerbi A, Vecchi A, et al. (2004) Increased survival, proliferation, and migration in metastatic human pancreatic tumor cells expressing functional CXCR4. Cancer Res 64: 8420-8427.

38. Decensi A, Veronesi U, Miceli R, Johansson H, Mariani L, et al. (2003) Relationships between plasma insulin-like growth factor I and insulinlike growth factor binding protein 3 and second breast cancer risk in a prevention trial of Fenretinide. Clin Cancer Res 9: 4722-4729.

39. André F, Bachelot T, Campone M, Dalenc F, Perez-Garcia JM, et al. (2013) Targeting FGFR with dovitinib (TKI258): preclinical and clinical data in breast cancer. Clin Cancer Res 19: 3693-3702.
40. Trudel S, Stewart AK, Rom E, Wei E, Li ZH, et al. (2006) The inhibitory anti-FGFR3 antibody, PRO-001, is cytotoxic to t $(4 ; 14)$ multiple myeloma cells. Blood 107: 4039-4046.

41. Qing J, Du X, Chen Y, Chan P, Li H, et al. (2009) Antibody-based targeting of FGFR3 in bladder carcinoma and $t(4 ; 14)$-positive multiple myeloma in mice. J Clin Invest 119: 1216-1229.

42. Bai A, Meetze K, Vo NY, Kollipara S, Mazsa EK, et al. (2010) GP369, an FGFR2-IIIb-specific antibody, exhibits potent antitumor activity against human cancers driven by activated FGFR2 signaling. Cancer Res 70: 7630-7639.

43. Van Zweeden AA, van der Vliet HJ, Wilmink JW, Meijerink MR, Meijer OW, et al. (2015) Phase I Clinical Trial to Determine the Feasibility and Maximum Tolerated Dose of Panitumumab to Standard GemcitabineBased Chemoradiation in Locally Advanced Pancreatic Cancer. Clin Cancer Res 21: 4569-4575. 